\title{
Vibration of Initially Stressed Nonlocal Euler-Bernoulli Nano-Beams
}

\author{
${ }^{* 1}$ Çiğdem Dinçkal \\ ${ }^{1}$ Çankaya University, Faculty of Engineering, Department of Civil Engineering, Ankara, Turkey \\ Received: 2017-06-16 Accepted: 2018-01-21
}

\begin{abstract}
This paper is pertinent to the analytical solutions for vibration analysis of initially stressed Nonlocal Euler-Bernoulli nano-beams. In order to take into account of small length scale effect, this vibration problem formulation is depending upon both nonlocal Euler-Bernoulli and also Eringen's nonlocal elasticity theory. The boundary conditions and governing equation are obtained by use of Hamiltonian's principle. These equations are solved analytically with different initial stresses (both compressive and tensile) and boundary conditions. The effect of small length scale and the initial stress on the fundamental frequency are investigated. The solutions obtained are compared with the ones depending upon both classical Euler-Bernoulli and Timoshenko beam theory to comprehend the responses of nanobeams under the effect of initial stress and small scale in terms of frequencies for both theories. The results supply a better declaration for vibration analysis of nano-beams which are short and stubby with initial stress.
\end{abstract}

Keywords: Nonlocal Euler-Bernoulli beam theory, fundamental frequency, initially stressed nano-beams, small length scale effect, nonlocal elasticity

\section{INTRODUCTION}

The recent advances in nanotechnology and microtechnology have paved the way for the manufacturing of all types of nano/micro-structures such as nanowires, nanoactuators, NEMS/MEMS devices and nanobeams[1-3].

The axial residual stress effects are usually imperative during the manufacturing of nano/micro- structures. Initial stresses are prevalent in the nano and micro scale systems on account of a high proportion of surface atoms. Initial stresses are existing in unstretched micro/nanostructures on thermal equilibration in molecular dynamics simulation of nano/micro-structures. In the analysis of these structures, Wang and $\mathrm{Hu}[4]$ demonstrated the inadequacy of classical beam theories in grasping the small scale effect in nano/micro-structures. This means that, these theories are insufficient to estimate the reduction in phase velocities of wave propagation in SWCNT when the wave number is so great. With consideration of the small scale effect, Eringen[5, 6, 7] proposed the theory of nonlocal continuum mechanics. This theory is employed for modifying the beam theory for analysis of nano/micro-beams. In the nonlocal elasticity theory, the small-scale effects are seized by presuming that the stress at a point is a function of the strains at all points in the domain[5, 6, 7]. This is not valid in classical elasticity theory. Nonlocal theory regards long-range inter-atomic interaction and gives results dependent on the size of a body. Furthermore the sizedependent phenomena can be considerably explained by the nonlocal elasticity theory. The nonlocal elasticity theory for vibration, the bending, and buckling analyses in nano/micro-electromechanical devices have been used in some studies[8-15]. Some researchers have employed the Timoshenko beam theory in analyzing vibration of nano-beams

[16-21]. Despite, few studies have considered the initial stress effects in the analysis of vibration behavior of micro/nano-beams[22-24]. Only Timoshenko beam theory has been used in these studies. Furthermore some researchers [25-31] have employed finite element as a tool in analyzing the effects of vibration of many structures. Neither of these studies has obtained analytical solutions for vibration of nano-beams based on nonlocal EulerBernoulli beam theory with considering both effects of initial stress and small length scale for all boundary conditions.

Thus, the present work aims to find out analytical solutions for the vibration of nano/micro initially stressed (compressive or tensile) beam depending upon Eringen's nonlocal elasticity and nonlocal Euler-Bernoulli beam theory by taking into account of both initial stress (such as compressive and tensile) and small scale effect.

The governing equation and boundary conditions are attained from Hamiltonian's principle.

The exact fundamental frequencies for nano-beams are attained by taking into consideration both initial stress and small length scale effect.

\section{MATERIALS AND METHOD}

The strain-displacement relation is explained by the following expression in Euler-Bernoulli theory[4, 5, 7, 19]

$$
\varepsilon_{x x}=-z \frac{d^{2} w}{d x^{2}}
$$

*Sorumlu Yazar: Çankaya University, Faculty of Engineering, Department of Civil Engineering, Ankara, Turkey cigdemdinckal2004@yahoo.com 
where, $z$ the coordinate which is measured from the midplane of the beam, $\boldsymbol{x}$ the longitudinal coordinate which is measured from the left end of the beam, $\boldsymbol{\varepsilon}_{\boldsymbol{x} x}$ is the normal strain and $\mathbf{w}$ is the transverse displacement. The virtual strain energy $\boldsymbol{\delta} \boldsymbol{U}$ is explained by the following integral

$\delta U=\iint_{0 A}^{L} \sigma_{x x} \delta \varepsilon_{x x} d A d x$

where, $\boldsymbol{L}$ is the length of the beam, $\boldsymbol{\sigma}_{x x}$ the normal stress, and $A$ the cross-sectional area. By putting Eq. (1) into Eq. (2), the strain energy can be represented by

$\delta U=-\int_{0}^{L} M \frac{d^{2} \delta w}{d x^{2}} d x$

here the bending moment $\boldsymbol{M}$ is stated by

$M=\int_{A} \sigma_{x x} z d A$

When a compressive initial stress $\sigma_{\boldsymbol{o}}$ applied to a beam, the virtual potential energy $\boldsymbol{\delta} \boldsymbol{V}$ of the initial stress is expressed as

$\delta V=-\int_{0}^{L} \sigma_{o} A \frac{d \delta w}{d x} \frac{d w}{d x} d x$

$\boldsymbol{\sigma}_{\boldsymbol{o}}$ refers to tensile initial stress when it is negative. By supposing that motion is free harmonic, the virtual kinetic energy $\boldsymbol{\delta} \boldsymbol{T}$ is represented by

$\delta T=\int_{0}^{L} \rho A w \omega^{2} \delta w d x$

where, $\boldsymbol{\rho}$ is the mass density of the beam material and $\omega$ is the circular frequency. To Hamiltonian's principle, one can obtain

$\boldsymbol{\delta}(\boldsymbol{U}+\boldsymbol{V}-\boldsymbol{T})=\mathbf{0}$

$\delta(U+V-T)=$

$\int_{0}^{L}\left(-M \frac{d^{2} \delta w}{d x^{2}}-\sigma_{o} A \frac{d \delta w}{d x} \frac{d w}{d x}-\rho A \omega^{2} w \delta w\right) d x$

The following equation is obtained by applied integration by parts

$\int_{0}^{L}\left(\frac{d^{2} M}{d x^{2}}-\sigma_{o} A \frac{d^{2} w}{d x^{2}}+\rho A \omega^{2} w\right) \delta w d x+\left[M \frac{d \delta w}{d x}-\right.$

$\left.\frac{d M}{d x} \delta w+\sigma_{o} A \frac{d w}{d x} \delta w\right]_{0}^{L}=0$

Hence $\boldsymbol{\delta} \boldsymbol{w}$ is arbitrary in $\boldsymbol{0}<\boldsymbol{x}<\boldsymbol{L}$, the governing equation of motion may be derived as follows

$\frac{d^{2} M}{d x^{2}}=\sigma_{o} A \frac{d^{2} w}{d x^{2}}-\rho A \omega^{2} w$

Based upon Eq. (8), nonlocal Euler-Bernoulli beam theory's boundary conditions are

$w=0 \quad$ or $-\frac{d M}{d x}+\sigma_{o} A \frac{d w}{d x}=0$

$\frac{d w}{d x}=\operatorname{0or} M=0$

Either one of these conditions may be stated. In one dimensional case, for an elastic material, Eringen's complicated nonlocal constitutive relation may be rewritten as[7]

$\sigma_{x x}-\left(e_{0} a\right)^{2} \frac{d^{2} \sigma_{x x}}{d x^{2}}=E \varepsilon_{x x}$

Where, $\boldsymbol{e}_{\mathbf{0}}$ is a constant determined experimentally or by calibrating with atomistic modeling, $\boldsymbol{e}_{\mathbf{0}} \boldsymbol{a}$ is the scale coefficient in which $a$ is the internal characteristic length (e.g., granular size, molecular diameter and lattice parameter) and $E$ is the Young's modulus. By multiplying
Eq. (12) by $z d A$ and integrating the results over the area $A$ gives

$M-\left(e_{0} a\right)^{2} \frac{d^{2} M}{d x^{2}}=-E I \frac{d^{2} w}{d x^{2}}$

where $I$ is the moment of inertia. By inserting the Eq. (9) into Eq. (13), the following equation can be obtained

$M=-E I \frac{d^{2} w}{d x^{2}}+\left(e_{0} a\right)^{2}\left(\sigma_{o} A \frac{d^{2} w}{d x^{2}}-\rho A \omega^{2} w\right)$

So, the governing equation for the vibration of micro/nano-beams depending upon nonlocal EulerBernoulli theory is stated by

$\left(E I-\left(e_{0} a\right)^{2} \sigma_{o} A\right) \frac{d^{4} w}{d x^{4}}+\left(\left(e_{0} a\right)^{2} \rho A \omega^{2}+\sigma_{o} A\right) \frac{d^{2} w}{d x^{2}}-$
$\rho A \omega^{2} w=0$

\section{FINDINGS}

To ease the governing equation, the following nondimensional terms are defined as follows:

$\overline{\boldsymbol{x}}=\frac{\boldsymbol{x}}{\boldsymbol{L}} \quad$ and $\quad \overline{\boldsymbol{w}}=\frac{\boldsymbol{w}}{\boldsymbol{L}}$

Frequency parameter is denoted by

$\lambda=\sqrt{\omega^{2} \frac{\rho A L^{4}}{E I}}$

Scaling effect parameter is represented by

$\alpha=\frac{e_{0} a}{L}$

In classical Euler-Bernoulli theory, scaling effect parameter is 0 .

Initial stress parameter is given by

$\Lambda=\sigma_{o} \frac{A L^{2}}{E I}$

Employing the parameters given in Eqs. (16)-( 19), Eq. (15) becomes

$B_{1} \frac{d^{4} \bar{w}}{d x^{4}}+B_{2} \frac{d^{2} \bar{w}}{d x^{2}}+B_{3} \bar{w}=0$

where the coefficients $\mathbf{B}_{\mathbf{i}}, i=1,2,3$ are represented in terms of scaling effect and initial stress parameters as follows

$B_{1}=1-\alpha^{2} \Lambda, B_{2}=\alpha^{2} \lambda^{2}+\Lambda, \quad B_{3}=-\lambda^{2}$

The general solution of Eq. (20) is

$\overline{\boldsymbol{w}}=c_{1} \operatorname{Cosh}\left[\beta \frac{x}{L}\right]+c_{2} \operatorname{Sinh}\left[\beta \frac{x}{L}\right]+c_{3} \operatorname{Cos}\left[\gamma \frac{x}{L}\right]+$

$c_{4} \operatorname{Sin}\left[\gamma \frac{x}{L}\right]$

here

$\beta=\frac{\sqrt{-\mathrm{B}_{2}+\sqrt{\mathrm{B}_{2}{ }^{2}-4 \mathrm{~B}_{1} \mathrm{~B}_{3}}}}{\sqrt{2 \mathrm{~B}_{1}}}, \quad \gamma=\frac{\sqrt{\mathrm{B}_{2}+\sqrt{\mathrm{B}_{2}{ }^{2}-4 \mathrm{~B}_{1} \mathrm{~B}_{3}}}}{\sqrt{2 \mathrm{~B}_{1}}}$

The coefficients $c_{1}-c_{4}$ can be found by considering the related boundary conditions.

\subsection{Simply Supported Nano-Beam}

Based on both Eqs. (10) and (11), for simply supported end, the boundary conditions are expressed as at $\overline{\boldsymbol{x}}=\mathbf{0}$ and $\bar{x}=\mathbf{1}$;

$\bar{w}=0, \frac{d^{2} \bar{w}}{d x^{2}}=M=0$

By enforcing the boundary conditions and substituting Eq. (24) into Eq. (22), one encounters an eigenvalue problem with use of the following matrix: 
$M=\left[\begin{array}{cccc}1 & 0 & 1 & 0 \\ \frac{\beta^{2}}{L^{2}} & 0 & -\frac{\gamma^{2}}{L^{2}} & 0 \\ \operatorname{Cosh}[\beta] & \operatorname{Sinh}[\beta] & \operatorname{Cos}[\gamma] & \operatorname{Sin}[\gamma] \\ \frac{\beta^{2} \operatorname{Cosh}[\beta]}{L^{2}} & \frac{\beta^{2} \operatorname{Sinh}[\beta]}{L^{2}} & -\frac{\gamma^{2} \operatorname{Cos}[\gamma]}{L^{2}} & -\frac{\gamma^{2} \operatorname{Sin}[\gamma]}{L^{2}}\end{array}\right]$

The eigenvalues are computed by equating the determinant of the matrix in Eq. (25) to zero. Hence the solution of the related characteristic equation yields the fundamental frequencies. The fundamental frequency formulation for simply supported beam is found as

$\lambda=\sqrt{\frac{n^{4} \pi^{4}\left(1-\alpha^{2} \Lambda\right)-n^{2} \pi^{2} \Lambda}{\left(1+n^{2} \pi^{2} \alpha^{2}\right)}}$

The relation between initial stress acting on a simply supported armchair nano-beam and fundamental frequency at different scale parameters $\boldsymbol{\alpha}$ is investigated in Figure 1.

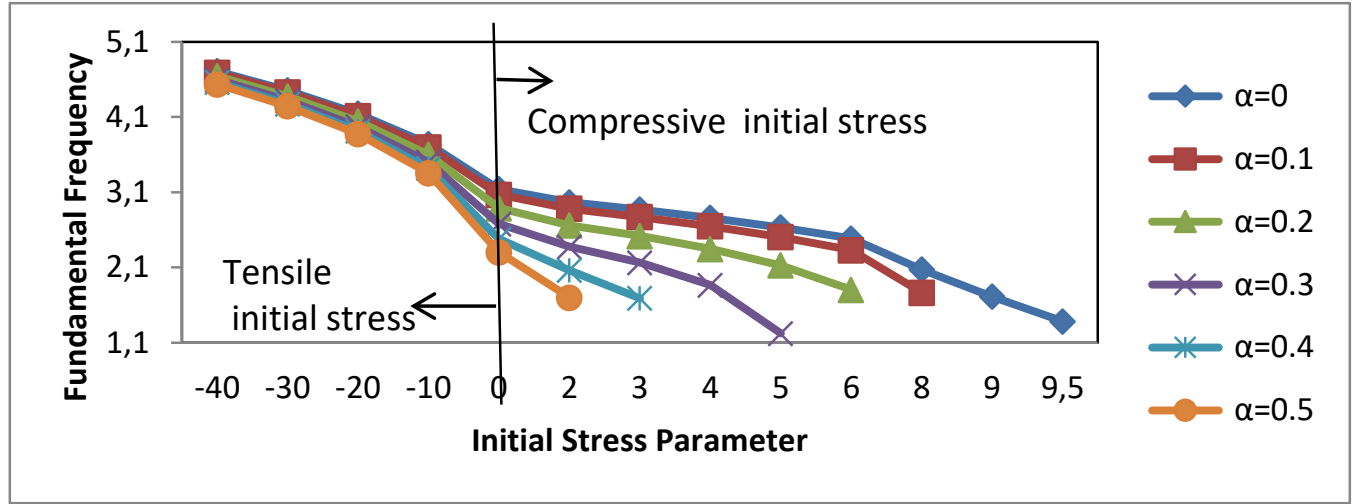

Figure 1. Small scale and initial stress effects on $\lambda$ for simply supported nano-beam

According to Figure 1, the existence of tensile initial stress and smaller scale parameter brings about an increase in the fundamental frequency. While compressive initial stress causes a decrease in fundamental frequency. Moreover an increase in scale effect (nonlocal) parameter causes a reduction in the frequencies.

To compare Timoshenko and nonlocal Euler and classical Euler theories in terms of fundamental frequency and initial stress parameter with different $\boldsymbol{\alpha}$ values, following figure is presented.

Table 1. Comparison of Timoshenko and Euler Theory with small scale and initial stress effects on $\lambda$ for simply supported nano-beam
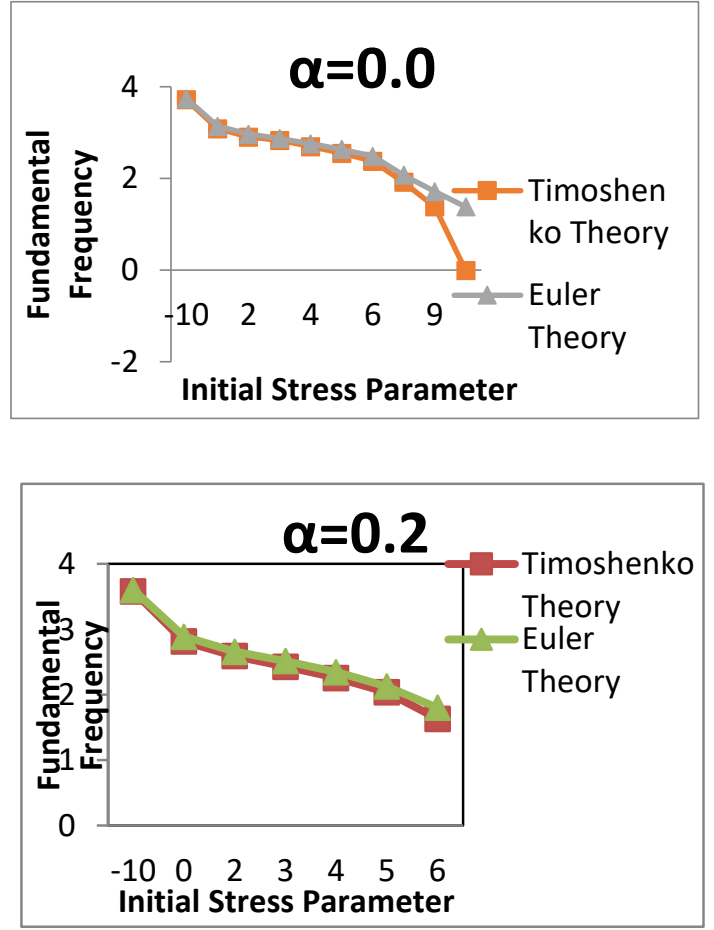
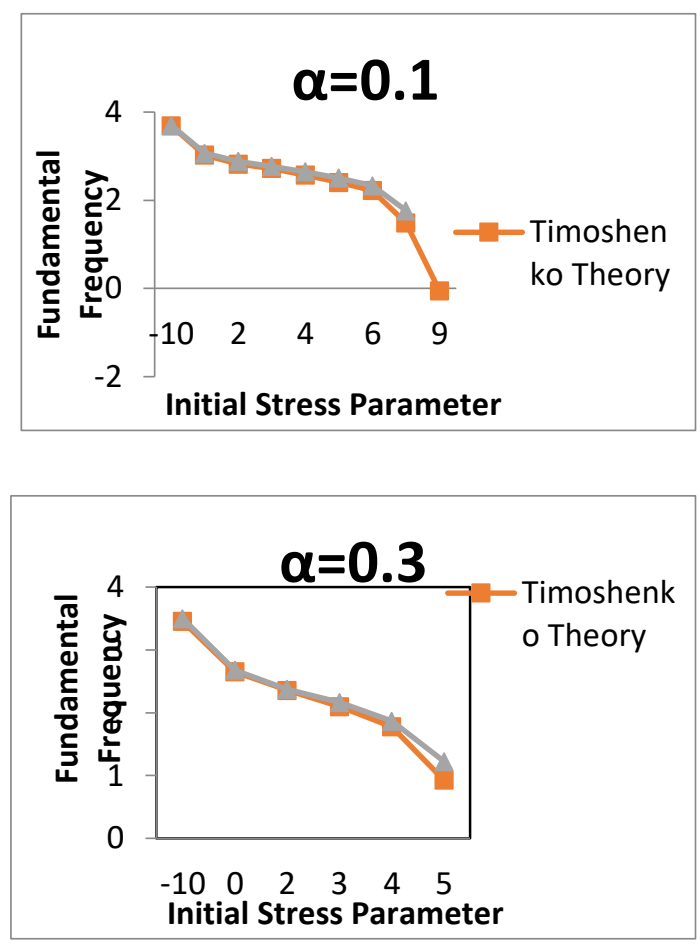
The frequency value increases with smaller scale effect in Euler theory than Timoshenko theory in Table 1. When $\boldsymbol{\alpha}$ value equals to 0 , classical Euler-Bernoulli beam theory is the case. It is also seen that the frequency values are almost same as tensile and compressive initial stress when $\boldsymbol{\Lambda}<5$ where there exists a critical stress of each $\boldsymbol{\alpha}$ value and vibration ceases.

Multiplying the general solution in Eq. (22) with the inverse of matrix in Eq. (25) gives a row vector $N$ which consists of four shape functions and each one corresponds to a unit value of the generalized frequency.

$N_{1}=\frac{\beta^{2} \operatorname{Csc}[\gamma] \operatorname{Sin}\left[\gamma-\frac{x \gamma}{L}\right]+\gamma^{2} \operatorname{Csch}[\beta] \operatorname{Sinh}\left[\beta-\frac{x \beta}{L}\right]}{\beta^{2}+\gamma^{2}}$

$N_{2}=\frac{L^{2}\left(-\operatorname{Csc}[\gamma] \operatorname{Sin}\left[\gamma-\frac{x \gamma}{L}\right]+\operatorname{Csch}[\beta] \operatorname{Sinh}\left[\beta-\frac{x \beta}{L}\right]\right)}{\beta^{2}+\gamma^{2}}$

$$
\begin{gathered}
N_{3}=\frac{\left(\beta^{2} \operatorname{Csc}[\gamma] \operatorname{Sin}\left[\frac{x \gamma}{L}\right]+\gamma^{2} \operatorname{Csch}[\beta] \operatorname{Sinh}\left[\frac{x \beta}{L}\right]\right)}{\beta^{2}+\gamma^{2}} \\
N_{4}=\frac{L^{2}\left(-\operatorname{Csc}[\gamma] \operatorname{Sin}\left[\frac{x \gamma}{L}\right]+\operatorname{Csch}[\beta] \operatorname{Sinh}\left[\frac{x \beta}{L}\right]\right)}{\beta^{2}+\gamma^{2}}
\end{gathered}
$$

To investigate only the length effect, one can take limits of these shape functions when $\beta$ and $\gamma$ converge to zero. Then Eqs. (27) to (30) take the following form:

$$
\begin{aligned}
& N_{1}=1-\frac{\mathrm{x}}{\mathrm{L}} \\
& N_{2}=-\frac{x\left(2 L^{2}-3 L x+x^{2}\right)}{6 \mathrm{~L}} \\
& N_{3}=\frac{x}{\mathrm{~L}} \\
& N_{4}=\frac{-L^{2} x+x^{3}}{6 \mathrm{~L}}
\end{aligned}
$$

Table 2 displays the shape functions for different lengths.

Table 2. Shape functions with various lengths for simply supported nano-beam
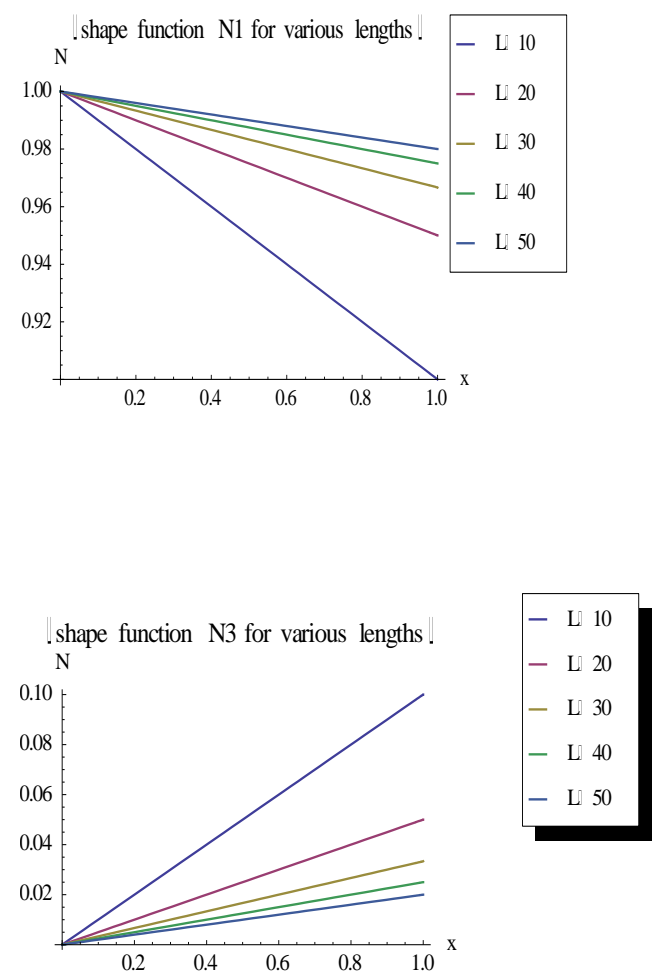

To Table 2, it is seen that an increase in length of the simply supported nano-beam leads to a decrease in $N_{2}, N_{3}$, $N_{4}$ corresponding to a unit value of the generalized frequency.

\subsection{Cantilever Nano-Beam}

Due to both Eqs. (10) and (11), for cantilever beam, the boundary conditions at $\bar{x}=0$ and $\bar{x}=1$ are

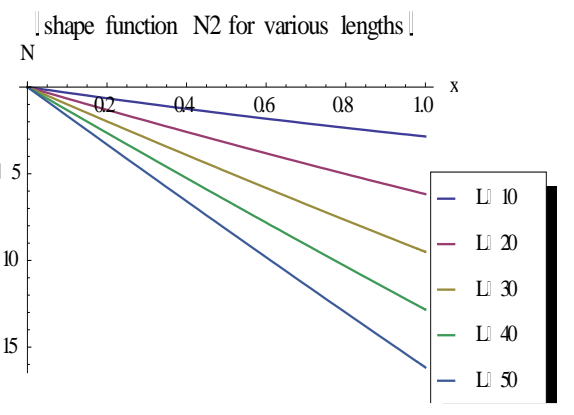

. shape function N4 for various lengths .

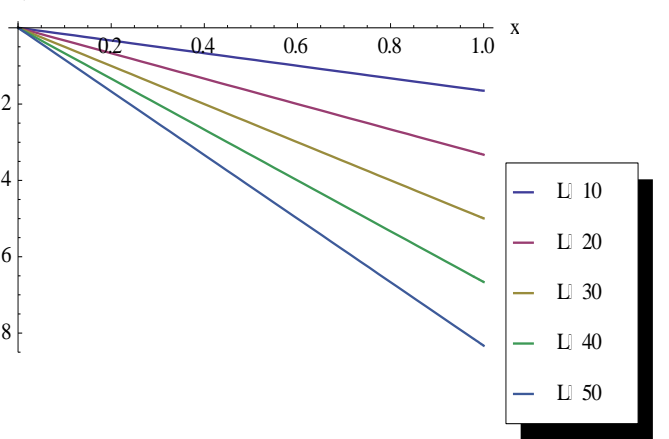

expressed as

$\bar{w}=0, \frac{d \bar{w}}{d x}=0$

$\frac{d^{3} \bar{w}}{d x^{3}}=0, \frac{d^{2} \bar{w}}{d x^{2}}=M=0$

By applying the boundary conditions and putting Eqs. (35) and (36) into Eq. (22), one needs to solve an eigenvalue problem with use of the following matrix: 
$M=\left[\begin{array}{cccc}1 & 0 & 1 & 0 \\ 0 & \frac{\beta}{L} & 0 & \frac{\gamma}{L} \\ \frac{\beta^{3} \operatorname{Sinh}[\beta]}{L^{3}} & \frac{\beta^{3} \operatorname{Cosh}[\beta]}{L^{3}} & \frac{\gamma^{3} \operatorname{Sin}[\gamma]}{L^{3}} & -\frac{\gamma^{3} \cos [\gamma]}{L^{3}} \\ \frac{\beta^{2} \cosh [\beta]}{L^{2}} & \frac{\beta^{2} \operatorname{Sinh}[\beta]}{L^{2}} & -\frac{\gamma^{2} \cos [\gamma]}{L^{2}} & -\frac{\gamma^{2} \operatorname{Sin}[\gamma]}{L^{2}}\end{array}\right]$

The fundamental frequency formulation for cantilever nano-beam is obtained as

$$
\lambda=\frac{\pi}{2} \sqrt{\frac{-4 \Lambda-\pi^{2}}{\left(4+\pi^{2} \alpha^{2}\right)}}
$$

The eigenvalues are calculated by equating the determinant of the matrix in Eq. (37) to zero. Solution of the related characteristic equation gives the fundamental frequencies. These frequencies are computed for various initial stresses and scale effect parameters. The results are presented in Figure 2.

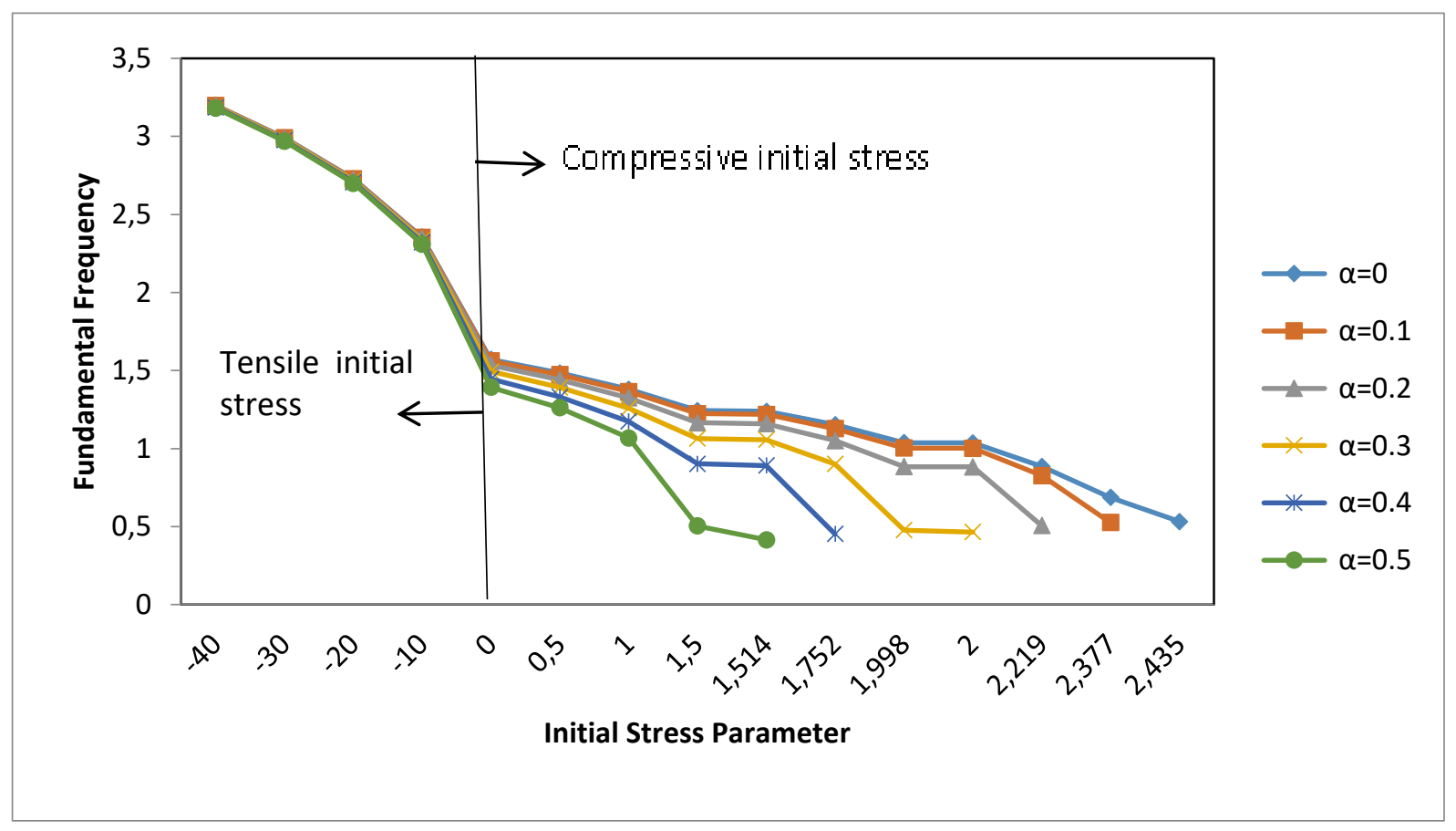

Figure 2. Small scale and initial stress effects on $\lambda$ for cantilever nano-beam

To Figure 2, it is obvious that the existence of tensile initial stress and smaller scale parameter bring on an increase in $\lambda$. While compressive initial stress causes a decrease in fundamental frequency. While small length scale increases, fundamental frequencies decrease. This is also seen in Table 3.

Timoshenko and Euler theories both classical and nonlocal in terms of fundamental frequency and initial stress parameter are compared at different scale effect parameters. As a result the following figures in Table 3 are plotted. 
Table 3. Comparison of Timoshenko and Euler Theory with small scale and initial stress effects on $\lambda$ for cantilever nano-beam
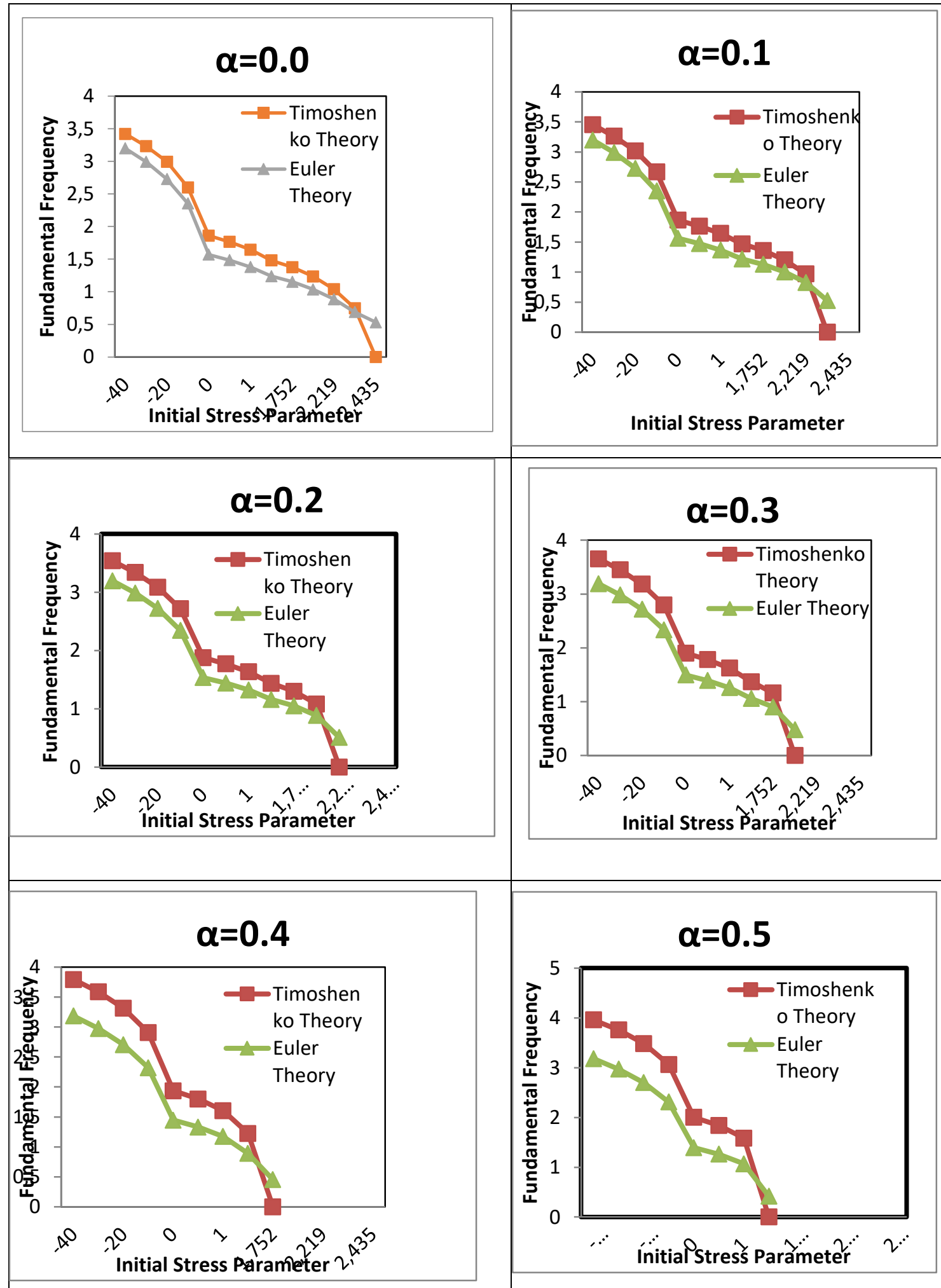

To Table 3, the frequency value increases when initial stress is tensile and theory is Timoshenko. With $\boldsymbol{\Lambda}<2.5$, the frequency values become zero in Timoshenko Theory

where there exists a critical stress of each $\boldsymbol{\alpha}$ value and vibration ceases. It is also observed that the frequency values are almost same in higher scale effects. 
Multiplying the general solution in Eq. (22) with the

consists of four shape functions and each one corresponds inverse of matrix in Eq. (37) gives a row vector $N$ which to a unit value of the generalized frequency for cantilever nano-beam.

$$
\begin{aligned}
& N_{1}=\frac{\left(\beta^{4} \operatorname{Cos}\left[\frac{x \gamma}{L}\right]+\gamma\left(\gamma^{2} \operatorname{Cosh}\left[\frac{x \beta}{L}\right](\gamma+\beta \operatorname{Sin}[\gamma] \operatorname{Sinh}[\beta])-\beta^{2} \operatorname{Sinh}[\beta]\left(\beta \operatorname{Sin}\left[\gamma-\frac{x \gamma}{L}\right]+\gamma \operatorname{Cos}[\gamma] \operatorname{Sinh}\left[\frac{x \beta}{L}\right]\right)+\right.\right.}{\left.\left.\beta \gamma \operatorname{Cosh}[\beta]\left(\beta \operatorname{Cos}\left[\gamma-\frac{x \gamma}{L}\right]+\beta \operatorname{Cos}[\gamma] \operatorname{Cosh}\left[\frac{x \beta}{L}\right]-\gamma \operatorname{Sin}[\gamma] \operatorname{Sinh}\left[\frac{x \beta}{L}\right]\right)\right)\right)} \\
& N_{2}=\frac{\left.\left.\left.\operatorname{Cos}[\gamma] \operatorname{Cosh}\left[\frac{x \beta}{L}\right]\right) \operatorname{Sinh}[\beta]\right)+L \gamma^{4}+\beta \gamma\left(2 \beta \gamma \operatorname{Cos}[\gamma] \operatorname{Cosh}[\beta]+\left(-\beta^{2}+\gamma^{2}\right) \operatorname{Sin}[\gamma] \operatorname{Sinh}[\beta]\right)\right)}{\left(\beta \gamma\left(\beta^{4}+\gamma^{4}+\beta \gamma\left(2 \beta \gamma \operatorname{Cos}[\gamma] \operatorname{Cosh}[\beta]+\left(-\beta^{2}+\gamma^{2}\right) \operatorname{Sin}[\gamma] \operatorname{Sinh}[\beta]\right)\right)\right)} \\
& N_{3}=\frac{\left.\beta \gamma \operatorname{Cosh}\left[\frac{x \beta}{L}\right](\gamma \operatorname{Sin}[\gamma]+\beta \operatorname{Sinh}[\beta])+\gamma\left(\gamma^{2} \operatorname{Cos}[\gamma]+\beta^{2} \operatorname{Cosh}[\beta]\right) \operatorname{Sinh}\left[\frac{x \beta}{L}\right]\right)}{\beta \gamma\left(\beta^{4}+\gamma^{4}+\beta \gamma\left(2 \beta \gamma \operatorname{Cos}[\gamma] \operatorname{Cosh}[\beta]+\left(-\beta^{2}+\gamma^{2}\right) \operatorname{Sin}[\gamma] \operatorname{Sinh}[\beta]\right)\right)} \\
& N_{4}=\frac{\left.\left.\operatorname{Cos}[\gamma] \operatorname{Cosh}\left[\frac{x \beta}{L}\right]\right)+\beta^{4} \operatorname{Sin}\left[\frac{x \gamma}{L}\right] \operatorname{Sinh}[\beta]+\gamma\left(\gamma^{3} \operatorname{Sin}[\gamma]-\beta^{3} \operatorname{Sinh}[\beta]\right) \operatorname{Sinh}\left[\frac{x \beta}{L}\right]\right)}{\beta \gamma\left(\beta^{4}+\gamma^{4}+\beta \gamma\left(2 \beta \gamma \operatorname{Cos}[\gamma] \operatorname{Cosh}[\beta]+\left(-\beta^{2}+\gamma^{2}\right) \operatorname{Sin}[\gamma] \operatorname{Sinh}[\beta]\right)\right)}
\end{aligned}
$$

To observe only the length effect, one can take limits of these shape functions when $\beta$ and $\gamma$ converge to zero. Then Eqs. (39) to (42) take the following form:

$N_{1}=1$

$N_{2}=x$

$N_{3}=\frac{1}{6} x^{2}(-3 L+x)$

$N_{4}=\frac{x^{2}}{2}$

Since only $N_{3}$ is the shape function dependent to length. $N_{3}$ is plotted for various lengths in Figure 3.

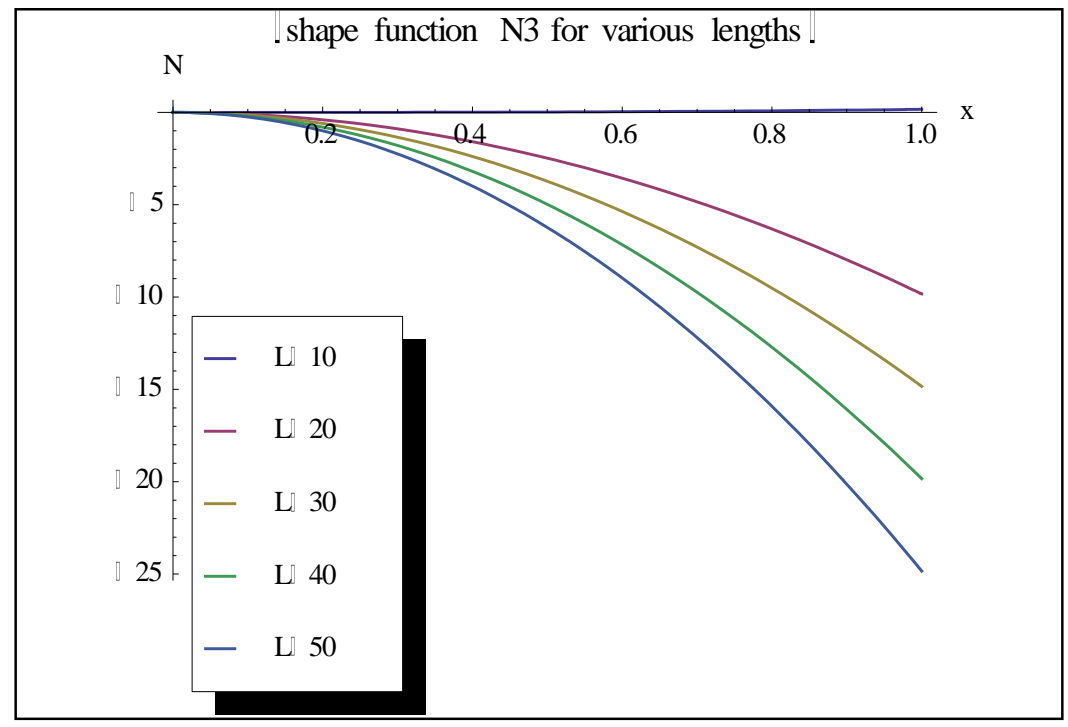

Figure 3. Shape function $N_{3}$ with various lengths for cantilever nano-beam

Figure 3 illustrates an increase in length values causes a reduction in related shape function.

\subsection{Clamped Nano-Beam}


By use of both Eqs. (10) and (11), for clamped beam, the boundary conditions at $\overline{\boldsymbol{x}}=\mathbf{0}$ and $\overline{\boldsymbol{x}}=\mathbf{1}$ are expressed as follows

$\bar{w}=0, \frac{d \bar{w}}{d x}=\mathbf{0}$

By enforcing the boundary conditions and substituting Eq. (47) into Eq. (22), an eigenvalue problem is required to solve with use of the following matrix:

$M=\left[\begin{array}{cccc}1 & 0 & 1 & 0 \\ 0 & \frac{\beta}{L} & 0 & \frac{\gamma}{L} \\ \operatorname{Cosh}[\beta] & \operatorname{Sinh}[\beta] & \operatorname{Cos}[\gamma] & \operatorname{Sin}[\gamma] \\ \frac{\beta \operatorname{Sinh}[\beta]}{L} & \frac{\beta \operatorname{Cosh}[\beta]}{L} & -\frac{\gamma \operatorname{Sin}[\gamma]}{L} & \frac{\gamma \operatorname{Cos}[\gamma]}{L}\end{array}\right]$

The eigenvalues are computed by equating the determinant of the matrix in Eq. (48) to zero. So $\lambda$ can be obtained due to the solution of the related characteristic equation. The computed $\lambda$ values are displayed under the effects of initial stresses and various scale effect parameters in Figure 4.

The fundamental frequency formulation for clamped nano-beam is computed as

$\lambda=\frac{2 \pi \sqrt{-\Lambda-2 \sqrt{2} \pi^{2}\left(-1+\alpha^{2} \Lambda\right)}}{\sqrt{\sqrt{2}+4 \pi^{2} \alpha^{2}}}$

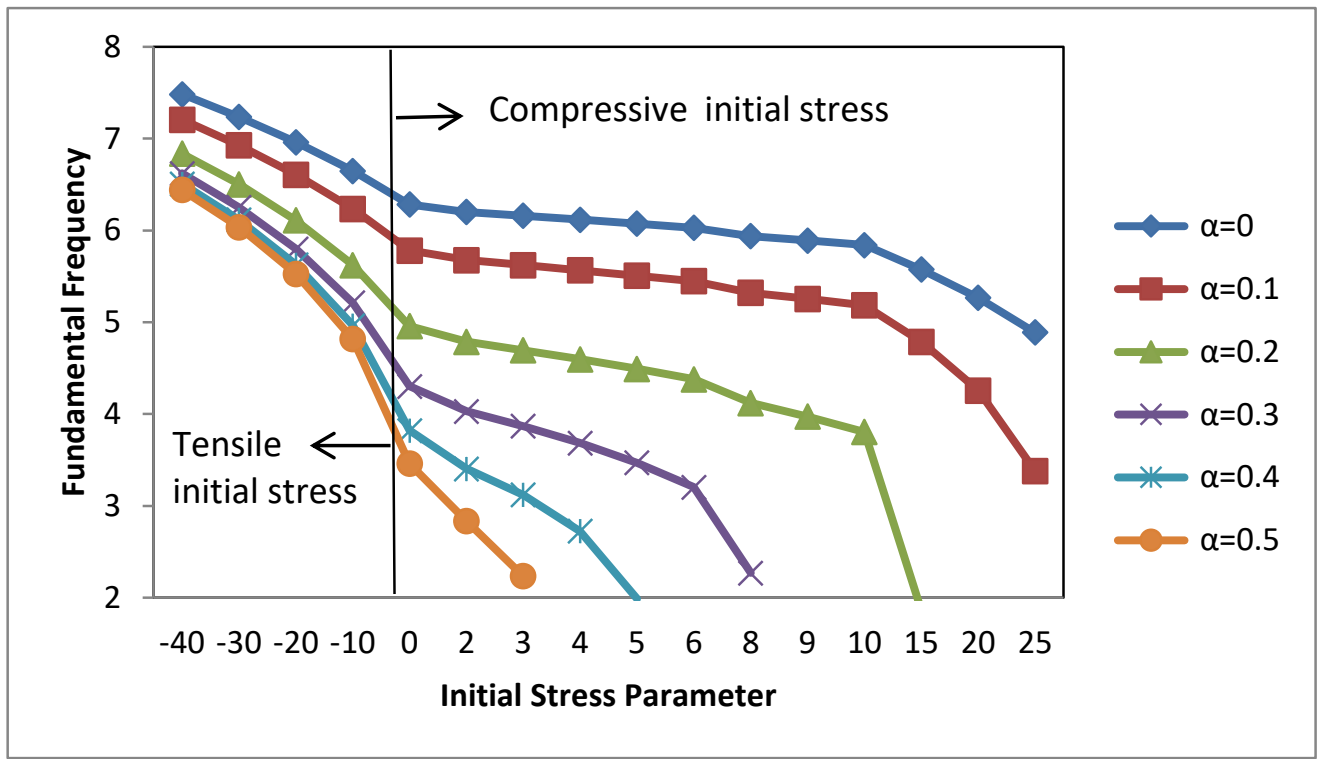

Figure 4. Small scale and initial stress effects on $\lambda$ for clamped nano-beam

According to Figure 4, smaller scale parameter and the existence of tensile initial stress bring on an increase in the fundamental frequency. Whereas compressive initial stress causes a decrease in fundamental frequency.

For clamped nano-beam, Timoshenko and nonlocal and classical Euler theories are compared with the effects of fundamental frequency and initial stress parameter at different scale effect parameters. As a result, Table 4 is presented. 
Table 4. Comparison of Timoshenko and Euler Theory with small scale and initial stress effects on $\lambda$ for clamped nano-beam
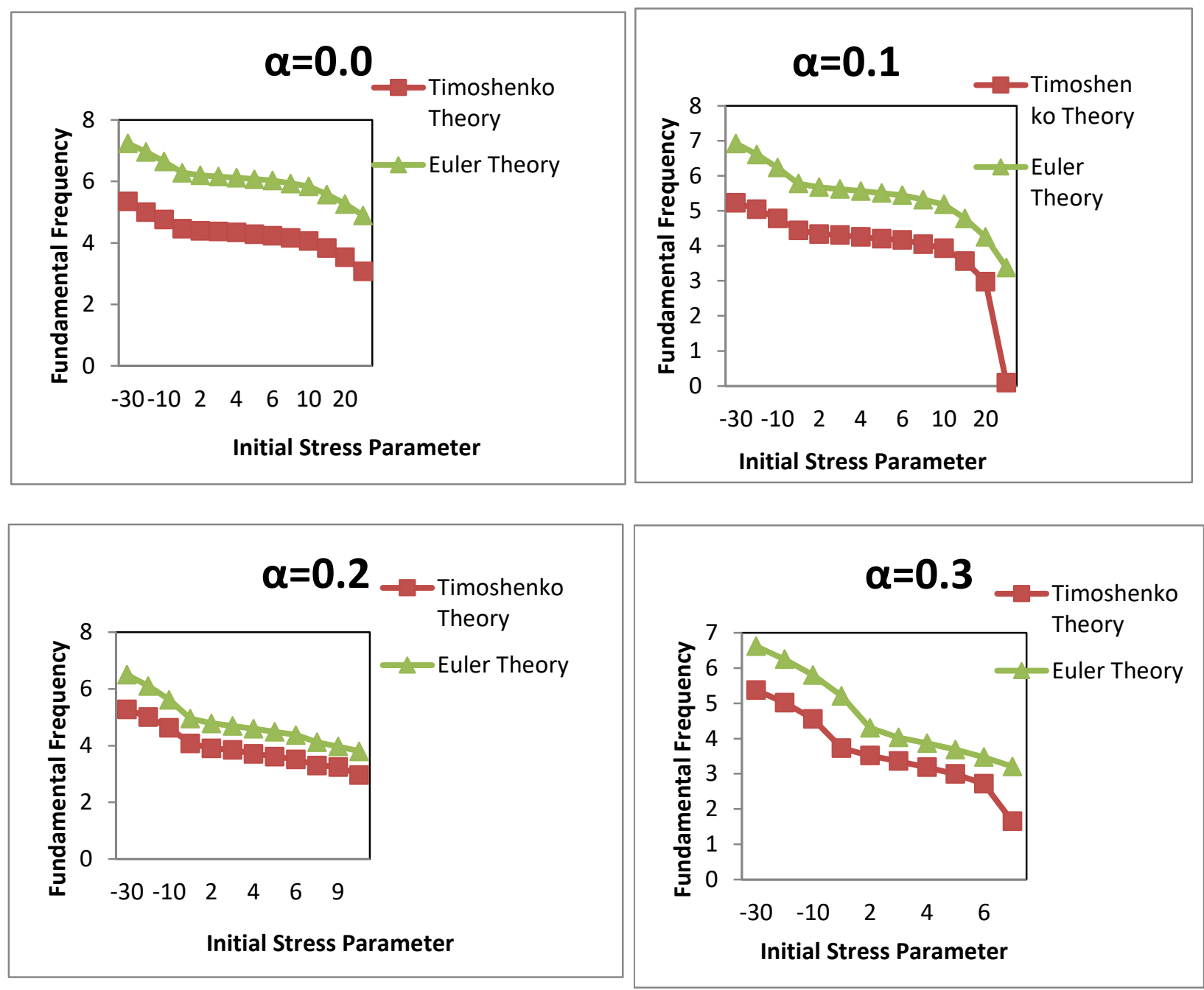

In Table 4, fundamental frequencies based on all theories display a decreasing trend when initial compressive stress and nonlocal parameter are increasing.

For clamped nano-beam, multiplying the general solution

in Eq. (22) with the inverse of matrix in Eq. (48) gives a row vector $N$ which consists of four shape functions and each one corresponds to a unit value of the generalized frequency.

$N_{1}=\frac{\beta \gamma \operatorname{Cos}\left[\frac{\gamma x}{L}\right]+\gamma \operatorname{Cosh}\left[\frac{\beta x}{L}\right]\left(\begin{array}{l}\beta-\gamma \operatorname{Sin}[\gamma] \operatorname{Sinh}[\beta])+\beta \operatorname{Sinh}[\beta]\left(\beta \operatorname{Sin}\left[\gamma-\frac{x \gamma}{L}\right]+\gamma \operatorname{Cos}[\gamma] \operatorname{Sinh}\left[\frac{x \beta}{L}\right]\right) \\ +\gamma \operatorname{Cosh}[\beta]\left(-\beta\left(\operatorname{Cos}\left[\gamma-\frac{x \gamma}{L}\right]+\operatorname{Cos}[\gamma] \operatorname{Cosh}\left[\frac{x \beta}{L}\right]\right)+\gamma \operatorname{Sin}[\gamma] \operatorname{Sinh}\left[\frac{x \beta}{L}\right]\right.\end{array}\right)}{2 \beta \gamma-2 \beta \gamma \operatorname{Cos}[\gamma] \operatorname{Cosh}[\beta]+(\beta-\gamma)(\beta+\gamma) \operatorname{Sin}[\gamma] \operatorname{Sinh}[\beta]}$

$N_{2}=\frac{\left(\begin{array}{c}L\left(-\beta \operatorname{Cosh}[\beta] \operatorname{Cosh}\left[\frac{x \beta}{L}\right] \operatorname{Sin}[\gamma]+\beta \operatorname{Sin}\left[\frac{x \gamma}{L}\right]+\beta \operatorname{Cosh}[\beta] \operatorname{Sin}\left[\gamma-\frac{x \gamma}{L}\right]-\gamma \operatorname{Cos}\left[\gamma-\frac{x \gamma}{L}\right] \operatorname{Sinh}[\beta]+\right. \\ \left.\gamma \operatorname{Cos}[\gamma] \operatorname{Cosh}\left[\frac{x \beta}{L}\right] \operatorname{Sinh}[\beta]+(\gamma-\gamma \operatorname{Cos}[\gamma] \operatorname{Cosh}[\beta]+\beta \operatorname{Sin}[\gamma] \operatorname{Sinh}[\beta]) \operatorname{Sinh}\left[\frac{x \beta}{L}\right]\right)\end{array}\right)}{2 \beta \gamma-2 \beta \gamma \operatorname{Cos}[\gamma] \operatorname{Cosh}[\beta]+(\beta-\gamma)(\beta+\gamma) \operatorname{Sin}[\gamma] \operatorname{Sinh}[\beta]}$

$N_{3}=\frac{\left.+\operatorname{Sin}\left[\frac{x \gamma}{L}\right](\gamma \operatorname{Sin}[\gamma]+\beta \operatorname{Sinh}[\beta])\right)-\gamma(\gamma \operatorname{Sin}[\gamma]+\beta \operatorname{Sinh}[\beta]) \operatorname{Sinh}\left[\frac{x \beta}{L}\right]}{2 \beta \gamma-2 \beta \gamma \operatorname{Cos}[\gamma] \operatorname{Cosh}[\beta]+(\beta-\gamma)(\beta+\gamma) \operatorname{Sin}[\gamma] \operatorname{Sinh}[\beta]}$

$N_{4}=\frac{L\left(\operatorname{Cosh}\left[\frac{x \beta}{L}\right](\beta \operatorname{Sin}[\gamma]-\gamma \operatorname{Sinh}[\beta])+\operatorname{Cos}\left[\frac{x \gamma}{L}\right](-\beta \operatorname{Sin}[\gamma]+\gamma \operatorname{Sinh}[\beta])+(\operatorname{Cos}[\gamma]-\operatorname{Cosh}[\beta])\left(\beta \operatorname{Sin}\left[\frac{x \gamma}{L}\right]-\gamma \operatorname{Sinh}\left[\frac{x \beta}{L}\right]\right)\right)}{2 \beta \gamma-2 \beta \gamma \operatorname{Cos}[\gamma] \operatorname{Cosh}[\beta]+(\beta-\gamma)(\beta+\gamma) \operatorname{Sin}[\gamma] \operatorname{Sinh}[\beta]}$ 
To see only the length effect, one can take limits of these shape functions when $\beta$ and $\gamma$ converge to zero for clamped beam. Then Eqs. (50) to (53) become

$$
\begin{aligned}
& N_{1}=\frac{(L-x)^{2}(L+2 x)}{L^{3}} \\
& N_{2}=\frac{(L-x)^{2} x}{L^{2}}
\end{aligned}
$$

$$
\begin{aligned}
& N_{3}=\frac{(3 L-2 x) x^{2}}{L^{3}} \\
& N_{4}=\frac{x^{2}(-L+x)}{L^{2}}
\end{aligned}
$$

Each of these new shape functions are plotted for various lengths in Table 5.

Table 5. Shape functions with various lengths for clamped nano-beam
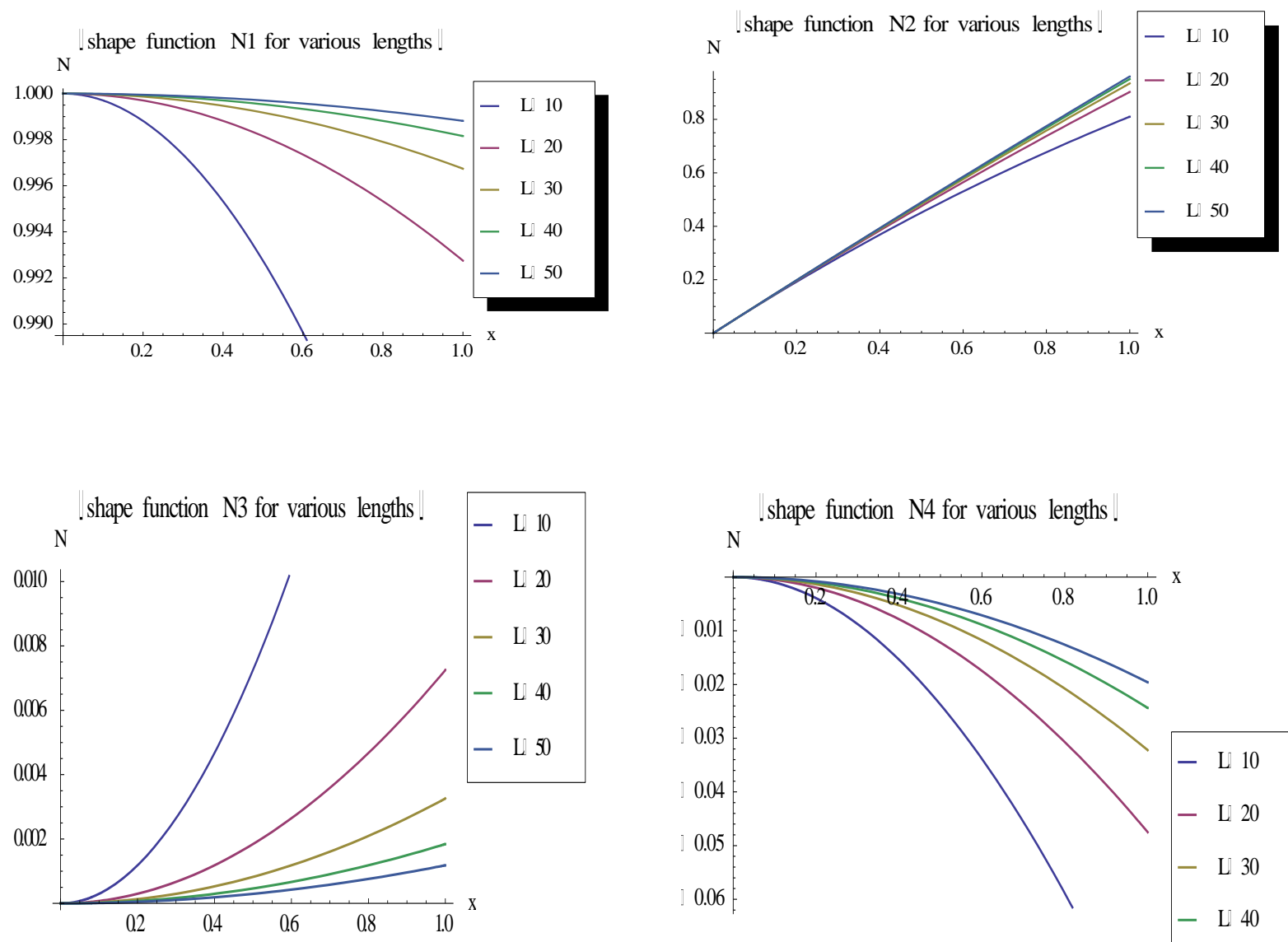

It is obvious from Table 5 that an increment in length of the clamped nano-beam leads to a decrease in $N_{3}, N_{4}$ corresponding to a unit value of the generalized frequency.

\subsection{Clamped-Simply Supported Nano-Beam}

Due to both Eqs. (10) and (11), the boundary conditions at $\overline{\boldsymbol{x}}=\mathbf{0}$ and $\overline{\boldsymbol{x}}=\mathbf{1}$ are represented as

$\bar{w}=\mathbf{0}, \frac{d \bar{w}}{d x}=\mathbf{0}$

$\bar{w}=0, \frac{d^{2} \bar{w}}{d x^{2}}=M=0$

By applying the boundary conditions and substituting

Eqs. (58) and (59) into Eq. (22), an eigenvalue problem is required to be solve with use of the following matrix:

$M=\left[\begin{array}{cccc}1 & 0 & 1 & 0 \\ 0 & \frac{\beta}{L} & 0 & \frac{\gamma}{L} \\ \operatorname{Cosh}[\beta] & \operatorname{Sinh}[\beta] & \operatorname{Cos}[\gamma] & \operatorname{Sin}[\gamma] \\ \frac{\beta^{2} \cosh [\beta]}{L^{2}} & \frac{\beta^{2} \operatorname{Sinh}[\beta]}{L^{2}} & -\frac{\gamma^{2} \operatorname{Cos}[\gamma]}{L^{2}} & -\frac{\gamma^{2} \operatorname{Sin}[\gamma]}{L^{2}}\end{array}\right]$

The eigenvalues are computed by equating the determinant of the matrix in Eq. (60) to zero. So the related characteristic equation can be solved to obtain the fundamental frequencies.

The fundamental frequency formulation for clamped simply supported nano-beam is computed as 
$\lambda=\frac{\sqrt{-\pi^{2} \Lambda-\pi^{4}\left(-1+\alpha^{2} \Lambda\right)}}{\sqrt{\left(1+\pi^{2} \alpha^{2}\right)}}$

The frequencies are illustrated in Figure 5 with initial stress parameters at various small scale effects.

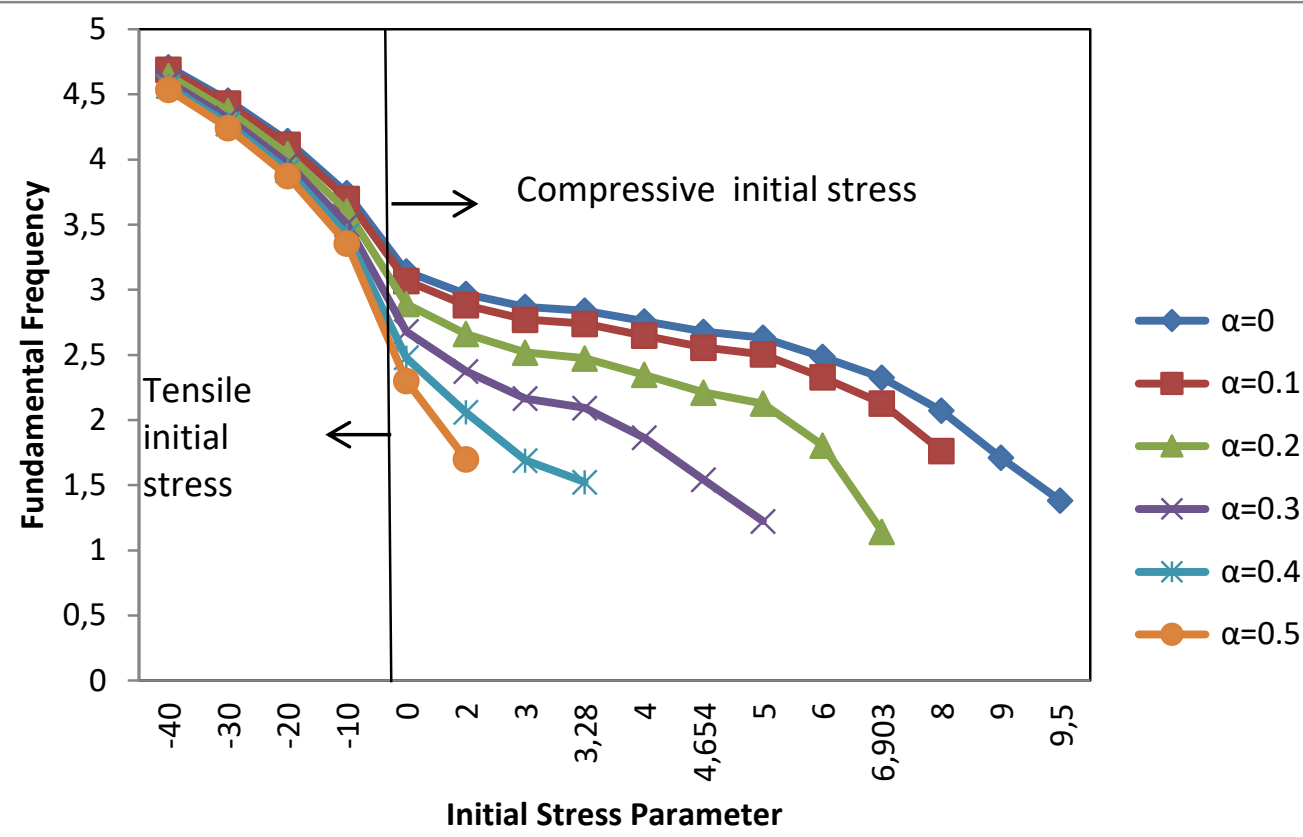

Figure 5. Small scale and initial stress effects on $\lambda$ for clamped-simply supported nano-beam

To Figure 5, smaller scale parameter and the existence of tensile initial stress bring on an increase in the fundamental frequency. While compressive initial stress and increasing scale effect cause a decrease in

fundamental frequency.

Timoshenko and both Euler theories are compared in terms of fundamental frequency and initial stresses at different scale effects in Table 6. 
Table 6. Comparison of Timoshenko and Euler Theory with small scale and initial stress effects on $\lambda$ of clampedsimply supported nano-beam
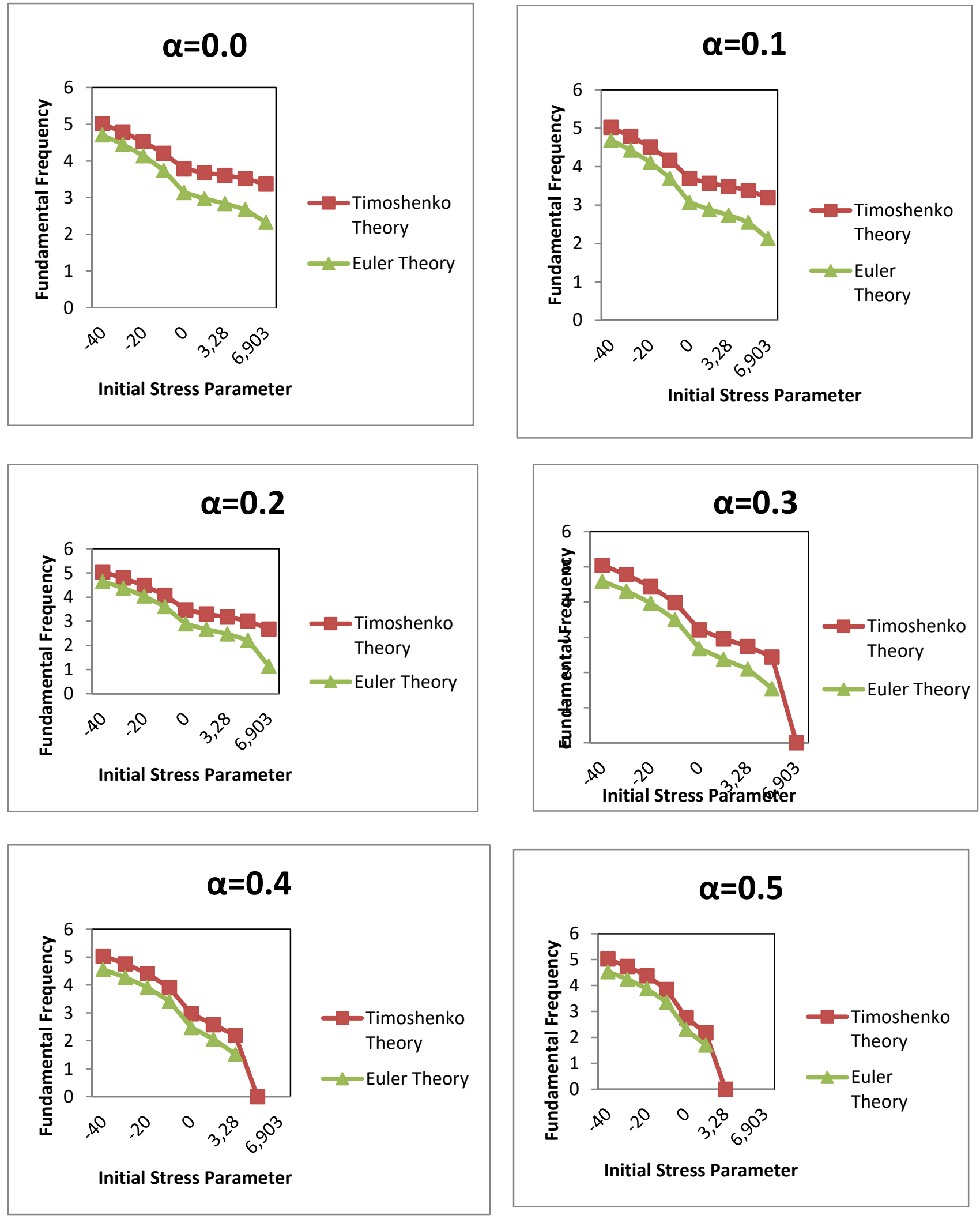
For clamped-simply supported nano-beam, Timoshenko Theory leads to an increase in the frequency values for all small effect parameters in Table 6. Furthermore, fundamental frequencies based on all theories display a decreasing trend when nonlocal parameter and initial compressive stress are increasing.

$N_{1}=\frac{-\gamma \operatorname{Cos}[\gamma] \operatorname{Cosh}\left[\frac{x \beta}{L}\right] \operatorname{Sinh}[\beta]+\operatorname{Cosh}[\beta]\left(\beta \operatorname{Sin}\left[\gamma-\frac{x \gamma}{L}\right]+\gamma \operatorname{Cos}[\gamma] \operatorname{Sinh}\left[\frac{x \beta}{L}\right]\right)}{\beta \operatorname{Cosh}[\beta] \operatorname{Sin}[\gamma]-\gamma \operatorname{Cos}[\gamma] \operatorname{Sinh}[\beta]}$

$N_{2}=\frac{\left(L\left(\left(-\operatorname{Cosh}\left[\frac{x \beta}{L}\right] \operatorname{Sin}[\gamma]+\operatorname{Sin}\left[\gamma-\frac{x \gamma}{L}\right]\right) \operatorname{Sinh}[\beta]+\operatorname{Cosh}[\beta] \operatorname{Sin}[\gamma] \operatorname{Sinh}\left[\frac{x \beta}{L}\right]\right)\right)}{\beta \operatorname{Cosh}[\beta] \operatorname{Sin}[\gamma]-\gamma \operatorname{Cos}[\gamma] \operatorname{Sinh}[\beta]}$ $\beta\left(\gamma^{2} \operatorname{Cos}[\gamma]+\beta^{2} \operatorname{Cosh}[\beta]\right) \operatorname{Sin}\left[\frac{x \gamma}{L}\right]-\beta \gamma \operatorname{Cos}\left[\frac{x \gamma}{L}\right](\gamma \operatorname{Sin}[\gamma]+\beta \operatorname{Sinh}[\beta])+$

$N_{3}=\frac{\beta \gamma \operatorname{Cosh}\left[\frac{x \beta}{L}\right](\gamma \operatorname{Sin}[\gamma]+\beta \operatorname{Sinh}[\beta])-\gamma\left(\gamma^{2} \operatorname{Cos}[\gamma]+\beta^{2} \operatorname{Cosh}[\beta]\right) \operatorname{Sinh}\left[\frac{x \beta}{L}\right]}{\left(\beta^{2}+\gamma^{2}\right)(\beta \operatorname{Cosh}[\beta] \operatorname{Sin}[\gamma]-\gamma \operatorname{Cos}[\gamma] \operatorname{Sinh}[\beta])}$

$N_{4}=\frac{L^{2}\left(\operatorname{Cosh}\left[\frac{x \beta}{L}\right](\beta \operatorname{Sin}[\gamma]-\gamma \operatorname{Sinh}[\beta])+\operatorname{Cos}\left[\frac{x \gamma}{L}\right](-\beta \operatorname{Sin}[\gamma]+\gamma \operatorname{Sinh}[\beta])+(\operatorname{Cos}[\gamma]-\operatorname{Cosh}[\beta])\left(\beta \operatorname{Sin}\left[\frac{x \gamma}{L}\right]-\gamma \operatorname{Sinh}\left[\frac{x \beta}{L}\right]\right)\right)}{\left(\beta^{2}+\gamma^{2}\right)(\beta \operatorname{Cosh}[\beta] \operatorname{Sin}[\gamma]-\gamma \operatorname{Cos}[\gamma] \operatorname{Sinh}[\beta])}$

To investigate only the length effect, one can take limits of these shape functions when $\beta$ and $\gamma$ converge to zero for clamped-simply supported nano-beam. Then Eqs. (62) to (65) take the following form:

$N_{1}=\frac{2 L^{3}-3 L x^{2}+x^{3}}{2 L^{3}}$

$N_{2}=\frac{x\left(2 L^{2}-3 L x+x^{2}\right)}{2 L^{2}}$

$N_{3}=\frac{x^{2}(3 \mathrm{~L}-\mathrm{x})}{2 L^{3}}$

$N_{4}=\frac{x^{2}(-\mathrm{L}+\mathrm{x})}{4 \mathrm{~L}}$

Each of these new shape functions are displayed at various lengths in Table 7.

Table 7. Shape functions with various lengths for clamped-simply supported nano-beam

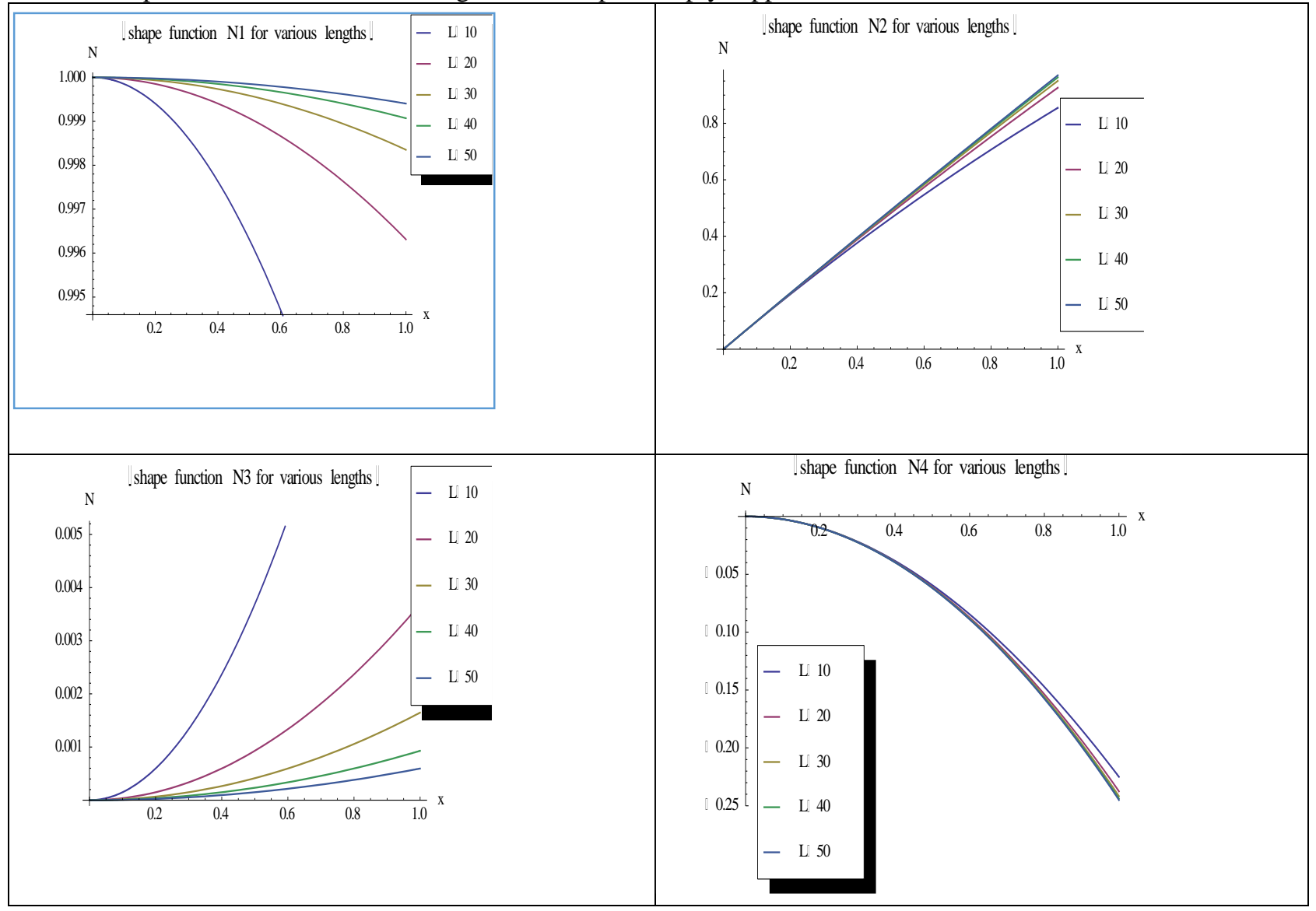


An increase in length of the clamped-simply supported nano-beam causes to a reduction in $N_{3}, N_{4}$ corresponding to a unit value of the generalized frequency.

\section{DISCUSSION AND CONCLUSIONS}

The boundary conditions and governing equation of motion for nano-beams dependent on Eringen's nonlocal elasticity and nonlocal Euler-Bernoulli theory are derived by use of Hamiltonian's principle with the consideration of small scale and initial stress effect. This beam formulation considers the existence of initial stress, nonlocal effect (small scale) and also relations of them with fundamental frequencies for all boundary conditions and this becomes remarkable when dealing with short and stubby beams such as nano-beams as the frequencies are high. In other words, for an increment in small scale (nonlocal) effect leads to a reduction in the value of fundamental frequencies. This decrease in fundamental frequency results from the incorporation of nonlocal effects, shear deformation parameter and mass inertia in the material properties of the initially stressed nanobeams. The nonlocal effect decreases the stiffness of the material and so the comparative lower fundamental frequencies.

Timoshenko and both nonlocal and classical Euler theories are also compared in terms of fundamental frequencies and initial stress at different small scale effects.

For all boundary conditions, existence of tensile initial stress leads to an increase in fundamental frequency values while compressive initial stress cause a decrease in frequency values. Moreover, the allowance for the small scale effect causes an increase in frequencies for all boundary conditions.

For simply supported and clamped nano-beam, Euler theory leads to an increase in frequencies only when the nonlocal parameter is not 0 so the case is not classical Euler theory while Timoshenko theory cause a reduction in frequencies for cantilever and clamped-simply supported nano-beam with various initial stress at different small scale effects.

As a result, this study yields physical perceptiveness which can be practical for the design and vibration analysis of micro/nano-structures.

Finally, this study is also practicable for acquiring the bending and buckling solutions of initially stressed micro/nano-beams using both Timoshenko and nonlocal Euler-Bernoulli beam theory. The present work can be furthered to analysis and design of nanostructures with complicated geometries and different load conditions beam theories for precise analysis of thick nano-structures based on both Timoshenko and nonlocal Euler-Bernoulli beam theory.

\section{REFERENCES}

[1] S. Iijima, "Nanotubes", Nature, vol. 354 pp. 56-58, 1999.

[2] J. Mongillo, Nanotechnology 101, London: Greenwood, 2009.

[3] M. Wilson, K. Kannangara, G. Smith, M. Simmons and B. Raguse, Nanotechnology, Basic

Science and Emerging Technologies, Australia:Chapman\&Hall/CRC, 2002.

[4] L. F. Wang and H. Y. Hu, "Flexural wave propagation in single-walled carbon nanotubes",

Phys. Rev. B., vol. 7, pp. 195412, 2005.

[5] A. C. Eringen, "Nonlocal polar elastic continua", Int. J. Eng. Sci., vol. 10, pp. 1-16, 1972.

[6] A. C. Eringen and D. Edelen, "On nonlocal elasticity", Int. J. Eng. Sci., vol. 10, pp. 233-248, 1972.

[7] A. C. Eringen, "On differential equations of nonlocal elasticity and solutions of screw

dislocation and surface waves", J. Appl. Phys., vol. 54, pp. 4703-4710, 1983.

[8] J. Peddieson, G. R. Buchanan and R. P. McNitt, "Application of nonlocal continuum models to nanotechnology", Int. J. Eng. Sci., vol. 41, pp. 305-312, 2003.

[9] L. J. Sudak, "Column buckling of multiwalled carbon nanotubes using nonlocal continuum

Mechanics", J. Appl. Phys., vol. 94, pp. 7281-7287, 2003. [10] Y. Q. Zhang, G. R. Liu and X. Y. Xie, "Transverse vibrations of double-walled carbon

nanotubes using a theory of nonlocal elasticity", Phys. Lett. A., vol. 340, pp. 258-266, 2005.

[11] Q. Wang, "Wave propagation in carbon nanotubes via nonlocal continuum mechanics", J. Appl. Phys., vol. 98, pp. 124301-124306, 2005.

[12] Q. Wang and V. K. Varadan, Vibration of carbon nanotubes studied using nonlocal

continuum mechanics, Smart. Mater. Struct., vol. 15, pp. 659-666, 2006.

[13] P. Lu, H. P. Lee, C. Lu and P. Q. Zhang, "Dynamic properties of flexural beams using a nonlocal elasticity model", J. Appl. Phys., vol. 99, pp. 073510, 2006.

[14] M. Xu, "Free transverse vibrations of nano-tomicron scale beams", Proceedings of the Royal Society A., vol. 462, pp. 2977-2995, 2006.

[15] B. I. Yakobson, C. J. Brabec and J. Bernholc, "Nanomechanics of carbon nanotubes:

Instability beyond linear response", Phys. Rev. Lett., vol. 76, pp. 2511-2514, 1996.

[16] C. M. Wang, Y. Y. Zhang and X. Q. He, "Vibration of nonlocal Timoshenko beams",

Nanotechnology, vol. 17, pp. 1-9, 2006. 
[17] Y. Y. Lee, C. M. Wang and S. Kitipornchai, "Vibration of Timoshenko beam with internal hinge", J. Eng. Mech., vol. 129, pp. 293-301, 2003.

[18] S. P. Timoshenko, Vibration Problems in Engineering, New York: Wiley, 1974.

[19] P. Prasad, "On the response of a Timoshenko beam under initial stress to a moving load", Int. J. Eng. Sci., vol. 19, pp. 615-628, 1981.

[20] J. Yoon, C. Q. Ru and A. Mioduchowski, "Timoshenko-beam effects on transverse wave propagation in carbon nanotubes", Compos. Part B-Eng., vol. 35, pp. 87-93, 2004.

[21] S. J. A. Koh and H. P. Lee, "Molecular dynamics simulation of size and strain rate dependent mechanical response of FCC metallic nanowires", Nanotechnology, vol. 17, pp. 3451-3467, 2006.

[22] T. Murmu and S. Adhikari, "Nonlocal elasticity based vibration of initially pre-stressed

coupled nanobeam systems", European Journal of Mechanics A/Solids, vol. 34, pp. 52-62, 2012.

[23] O. Rahmani, "On the flexural vibration of prestressed nanobeams based on a nonlocal theory", Acta Physica Polonica A, vol. 125, pp. 532-533, 2014.

[24] C. M. Wang, Y. Y. Zhang and S. Kitipornchai, "Vibration of initially stressed micro-and nano-beams", Int. J. Struct. Stab. Dy., vol. 7, pp. 555-570, 2007.
[25] B. N. Alemdar and P. Gülkan, "Beams on generalized foundations: supplementary element matrices", Eng. Struct., vol. 19, pp. 910-920, 1997.[26] J. K. Phadikar and S. C. Pradhan, "Variational formulation and finite element analysis for nonlocal elastic nanobeams and nanoplates", Comp. Mater. Sci., vol. 49, pp. 492-499, 2010.

[27] S. C. Pradhan, "Nonlocal finite element analysis and small scale effects of CNTs with

Timoshenko beam theory", Finite Elem. Anal. Des., vol. 50, pp. 8-20, 2012.

[28] S. Adhikari, T. Murmu and M. A. McCarthy, "Dynamic finite element analysis of axially vibrating nonlocal rods", Finite Elem. Anal. Des., vol. 63, pp. 42-50, 2013.

[29] Ç. Dinçkal, B. N. Alemdar and P. H. Gülkan, "Dynamics of a beam-column element on an elastic foundation", Can. J. Civ. Eng. vol. 43, pp. 685701, 2016.

[30] Ç. Dinçkal, Free vibration analysis of Carbon Nanotubes by using finite element method,

Iran. J. Sci. Technol. Trans. Mech. Eng., vol. 40, pp. 43$55,2016$.

[31] Ç. Dinçkal, "Finite element modeling for vibration of initially stressed nonlocal Euler-

Bernoulli beams", CBU J. of Sci., vol. 12, no 3, pp. 399411, 2016. 